\title{
Plasmon Resonances on Opto-capacitive Nanostructures
}

\author{
N. Shahcheraghi, A. Dowd, M.D. Arnold, and M.B. Cortie \\ Institute for Nanoscale Technology, University of Technology Sydney, Sydney, 2007, Australia \\ Email: michael.cortie@uts.edu.au
}

\begin{abstract}
Silver is considered as one of the most desirable materials for plasmonic devices due to it having low loss (low $\left.\varepsilon_{2}\right)$ across the visible spectrum. In addition, silver nanotriangles can self-assemble into complex structures that can include tip-totip or base-to-base arrangements. While the optical properties of tip-to-tip dimers of nanotriangles have been quite intensively studied, the geometric inverse, the base-to-base configuration, has received much less attention. Here we report the results of a computational study of the optical response of this latter configuration. Calculations were performed using the discrete dipole approximation. The effect of gap size and substrate are considered. The results indicate that the base-to-base configuration can sustain a strong coupled dipole and various multimode resonances. The pairing of the parallel triangle edges produces a strongly capacitive configuration and very intense electric fields over an extended volume of space. Therefore, the base-to-base configuration could be suitable for a range of plasmonic applications that require a strong and uniform concentration of electric field. Examples include refractometeric sensing or metal-enhanced fluorescence
\end{abstract}

Keywords: optical properties - plasmon resonance- silver nanotriangles

\section{INTRODUCTION}

The free electrons of nanoparticles of coinage metals $(\mathrm{Cu}, \mathrm{Ag}$, and $\mathrm{Au})$ and a few other elements are capable of undergoing strong resonant oscillations with the electric field of light at appropriate frequencies. The resulting coupled oscillation between photon and electrons is termed a localized surface plasmon resonance (LSPR). The LSPRs in metallic nanoparticles cause significant absorption and scattering of electromagnetic waves, and intense local electric field enhancement in the immediate vicinity of the particle surface. In general, the spectral position, damping, and strength of the resonance depend on the particle material, size, geometry, and the dielectric function of the surrounding host. ${ }^{1}$ These phenomena provide a wide range of opportunities for application in chemical and refractometric sensing and imaging $^{2}$, nanoscale optical devices, surface-enhanced spectroscopies (SERS) ${ }^{3}$, single molecule detection, metal enhanced fluorescence, enhancement of nonlinear optical effects and light guiding ${ }^{2}$ and even cancer-treatment ${ }^{4}$. An attractive aspect of using silver is that it is possible to produce a wide range of nanoparticle shapes by wet chemistry. These include nanotriangles which are useful because it has been demonstrated that electric field enhancement of nanoparticles with sharp tips is more powerful than that due to nanospheres owing to charge accumulating at the sharp corners $^{5,6}$. In addition, the in-plane dipole LPSRs of shapes like silver nanotriangles, nanoplates and nanoprisms can be tuned across the visible spectrum and into the near infrared. Therefore these anisotropic silver nanostructures potentially have a broad range of application in plasmonic devices. ${ }^{7}$ There is a strong dipolar LSPR and associated electromagnetic field enhancement when the lateral dimension of the silver nanoplate is larger than its thickness. A quadrupole plasmon excitation may also be observed, and additional multipolar resonances are reported for more complex arrangements of particles $^{8}$.

Nanotriangles of $\mathrm{Ag}$ can be prepared by solution chemistry ${ }^{9-11}$, electrochemical deposition ${ }^{12}$, nanosphere lithography ${ }^{13}$ or by some form of top-down lithography, such as electron beam lithography ${ }^{14}$ or focused ion beam milling ${ }^{15}$. Typical Ag nanotriangles produced by wet chemical synthesis are shown in Figure 1 and serve as inspiration for the prototypical geometries simulated in the present paper. Dimers of nanotriangles in the point-to-point (sometimes called 'nanobowtie ${ }^{16}$ ) configuration and in the head-to-tail configuration are known to exhibit large local E-field enhancement at socalled hot spots, and therefore have been already well-studied for their potential uses as optical antennas and waveguides $^{17}$ or for making surface-enhanced Raman measurements ${ }^{16}$. At least one multipolar resonance has been identified for the these configurations ${ }^{18}$, although the intensity of such a multipole resonance is generally much weaker than the dipole mode ${ }^{19}$. It is reported that the maximum field enhancement for the dipole resonance is at the triangle tips. 
In contrast, the significant regions of enhancement for the quadrupole resonance occur at the sides of the triangle, and the field decays away from the surface faster for the quadrupole than for the dipole 20

There has hitherto been far less interest in the base-to-base coupling of two nanotriangles, probably because it is widely believed that this configuration provides less useful amplification of the near-fields than the tip-to-tip or tip-to-base ones mentioned above. It is our hypothesis that this is an incorrect belief and that, for some applications, the base-to-base configuration will be competitive if not actually superior. The key point is that the volume in which electric field is enhanced is very much more extended than in the tip-to-tip or tip-to-base cases, and this compensates for the reduction in 'hot spot' intensity due to the tip(s). Our hypothesis should be valid for the case of refractometric and enhanced fluorescence sensing applications but it is probably not true for SERS applications (where the enhancement in signal is proportional to $E^{4}$ and hence hypersensitive to the peak value of the field intensity ${ }^{21}$ ). In the present work we examine the base-to-base configuration and confirm that it is a very versatile platform for developing both an extended volume of enhanced electric field strength and a range of interesting LSPRs.

The study described here exploits numerical simulations but, as we will show, the means to make well-formed Ag nanotriangles by wet chemical processes is now well-established. Therefore, we are confident that the optical phenomena that we will describe can be realized in real devices if required.

\section{METHODOLOGY}

Actual Ag nanotriangles were produced in order to verify the range of shapes that were feasible so that realistic examples of nanotriangles could be designed for the numerical simulations. A mixture of $\mathrm{AgNO}_{3}$ (purity $>99.8 \%$ ), $\mathrm{NaBH}_{4}$, polyvinylpyrrolidone (PVP), trisodium citrate $\left(\mathrm{Na}_{3} \mathrm{CA}>99.5 \%\right)$ and $\mathrm{H}_{2} \mathrm{O}_{2}(30 \mathrm{wt} \%)$ was used. The water used was high purity. An aqueous solution of $\mathrm{AgNO}_{3}(0.1 \mathrm{mM}, 25 \mathrm{ml})$, PVP $(0.7 \mathrm{mM}, 1.5 \mathrm{ml})$ and $\mathrm{NaBH}_{4}(100 \mathrm{mM}, 0.14 \mathrm{ml})$ was made and then $\mathrm{Na}_{3} \mathrm{CA}(30 \mathrm{mM}, 1.5 \mathrm{ml})$ was added. The solution was continuously stirred from the beginning of the synthesis which was conducted at room temperature. After several minutes, $\mathrm{H}_{2} \mathrm{O}_{2}(30 \mathrm{wt} \%, 0.06 \mathrm{ml})$ was injected into the solution. A few seconds later a colloid of triangular silver nanoparticles is formed. These give the liquid a bright blue color. Our recipe is a modified version of a procedure previously published by Tsuji et al. ${ }^{22}$ The optical properties of the resulting solutions were measured by UV-vis spectrometer to verify the position of the dipolar LPSR. Droplets of colloid were also dried out on sample mounts and imaged using scanning and transmission electron microscopy.

The optical properties of prototypical Ag nanotriangles measuring $80 \times 80 \times 80 \times 20 \mathrm{~nm}, 100 \times 100 \times 100 \times 20 \mathrm{~nm}$ and $100 \times 100 \times 100 \times 25 \mathrm{~nm}$ were simulated by the discrete dipole approximation (DDA) as implemented in the program DDSCAT $^{23,24}$. The basic approach of DDA is simulation of nanostructures as a cubic lattice of $N$ polarizable point dipoles and the response of this array of dipoles to an incident field are then determined numerically. The applicability and accuracy of this technique has been previously verified by various investigators ${ }^{20}$, and its particular advantage is that it can be applied to arbitrarily-shaped particles. The DDSCAT program has been generally found to give accurate results, provided that a sufficiently large number of closely spaced dipoles are used. However, while a $1 \mathrm{~nm}$ spacing of the dipoles in the DDA is sufficient for simple targets undergoing a dipole resonance, it is also known that simulations of more complex shapes with multipolar resonances or sharp protuberances may require an even finer dipole mesh in order to produce stable results. Therefore, in the present work we began by checking the effect of dipole spacing on the stability of our calculations. We found that a smaller dipole spacing generally increased the $\mathrm{Q}_{\text {ext }}$ associated with a given resonance peak, but that the qualitative nature and position of the various resonances was not changed.

There are basically three ways to excite a dipole resonance in a nanotriangle in vacuum, corresponding to applying the electric field in the through-thickness (short transverse) direction or in one of two possible in-plane (longitudinal) directions. The short transverse polarization is not very interesting in these targets and is hardly altered by the geometry; therefore our investigation will be directed at an analysis of the resonances produced by the longitudinal polarizations, Figure 1. The first of the in-plane possibilities has the electric field polarized parallel to an edge of a single triangle or parallel to the gap between two base-to-base triangles. The second possibility has the polarization perpendicular to an edge (single triangle) or to the gap (pair of triangles). We will consider both possibilities and designate the first as 'parallel polarization' and the second as 'perpendicular polarization'. In these nanoscale situations the optical extinction is controlled by direction of polarization and is not affected to any significant degree by the direction of propagation of the light. The calculated optical properties are expressed in terms of extinction efficiency, Qext. In the case of the DDSCAT code, this is the ostensible optical extinction cross-section normalized by the nominal geometric crosssectional area of the particle, expressed as if all the matter in the particle was packed into a sphere. The electric fields of the resonances were visualized with software written by one of the authors (M.B.C.). 


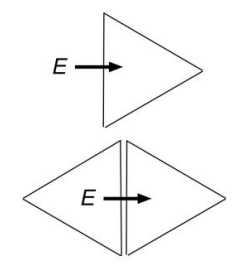

$\perp$ perpendicular

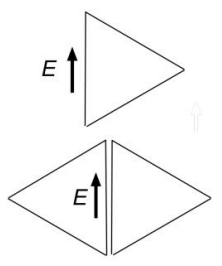

II parallel

Figure 1: Definition of the two types of longitudinal polarization applied in the present work.

\section{RESULTS AND DISCUSSION}

3.1 Characterization of experimental samples

Figure 2 provides SEM and TEM images of silver nanotriangles. The edge length of well-formed triangles is generally in the range of 60 to $100 \mathrm{~nm}$ and the thickness (visible when the triangles form stacks seen side-on, not shown here but previously discussed elsewhere ${ }^{25}$ ) in the range 15 to $30 \mathrm{~nm}$. Therefore, we chose prototypical triangular structures of $80 \times 80 \times 80 \times 20,100 \times 100 \times 100 \times 20 \mathrm{~nm}$ and $100 \times 100 \times 100 \times 25 \mathrm{~nm}$ for our numerical simulations. It is also apparent in these images that the triangles become arranged in various configurations during the drying process. The base-to-base configuration (arrowed) is one of these and is discussed here.
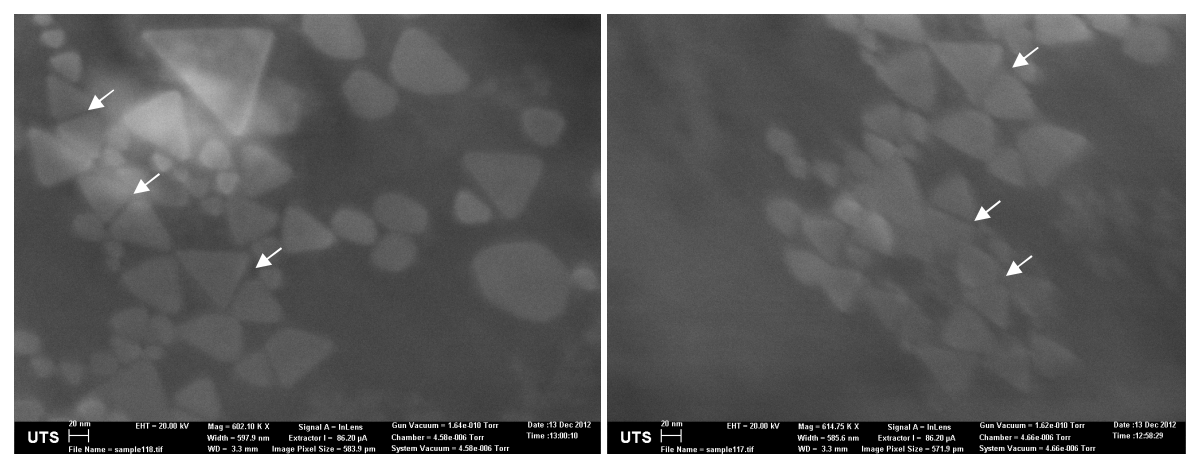


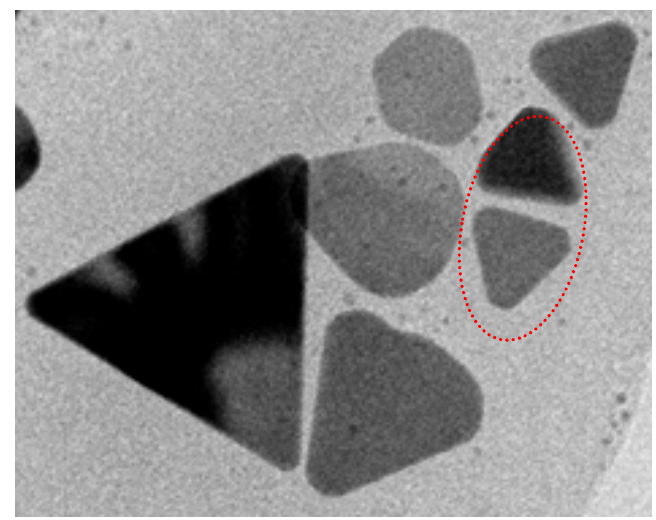

Figure 2: a) and b) SEM images with base-to-base configurations arrowed, c) TEM image of triangles, base-to-base configuration circled.

\subsection{Simulation of optical properties and electric field of nanotriangles}

The extinction efficiencies of a single nanotriangle and of a pair of nanotriangles that are nearly touching in the base-tobase configuration are shown in Figure 3. For simplicity we first consider the case where the triangles are in vacuum. It is clear that the spectral position of LSPRs of nanotriangles can be red-shifted by a large amount by placing them in dimer configurations.

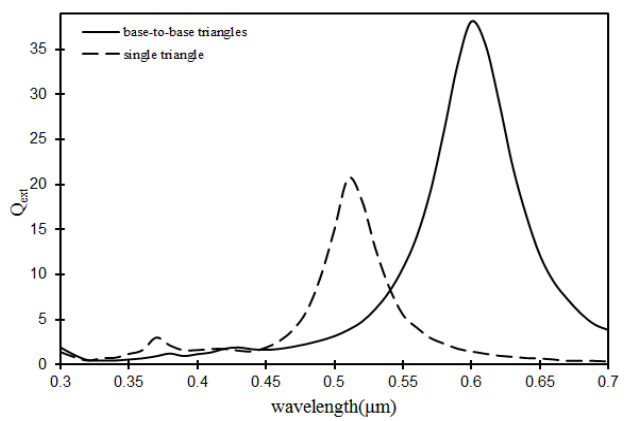

Figure 3. The calculated extinction spectra of $80 \times 80 \times 80 \times 20 \mathrm{~nm}$ silver triangles in the single and base-to-base $(0.7 \mathrm{~nm}$ gap $)$ configurations. 'Perpendicular polarization', as defined in the Methodology section, has been applied.

Next, we consider what happens when the base-to-base triangles are placed on a glass substrate. Figure 4 compares the extinction spectra for base-to-base triangles which are placed on a glass substrate to those without a substrate. In this example the gap is $5 \mathrm{~nm}$ and the simulated glass substrate measures $100 \times 100 \times 20 \mathrm{~nm}$. 'Perpendicular polarization' was applied as in the previous figure. It is clear that the primary effect of the substrate has been to red-shift the LSPR. The red-shifting has 'stretched' the absorption spectrum allowing a new resonance at about $475 \mathrm{~nm}$ to become apparent. This resonance may be related to the interface between the glass substrate and the underneath surface of the triangles. Note, however, that the 'attenuation' of $Q_{\text {ext }}$ when the substrate is present is largely a geometric artefact produced by the way in which $Q_{\text {ext }}$ is defined. 


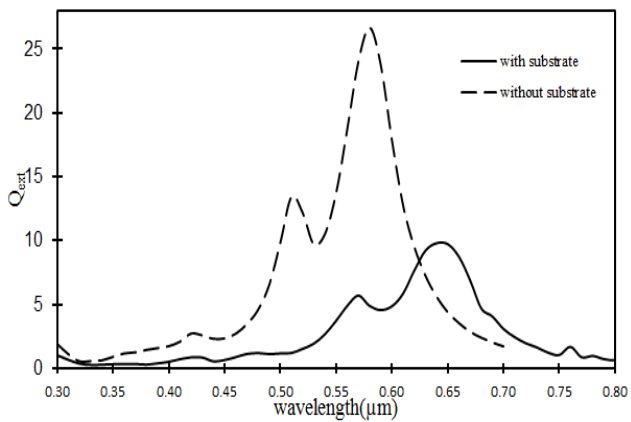

Figure 4.The calculated extinction spectra of $80 \times 80 \times 80 \times 20 \mathrm{~nm}$ silver nanotriangles on glass substrate and without substrate.

Having gained a general understanding of the behavior of base-to-base triangles on a glass substrate, we then compared the effect of the two kinds of in-plane polarization, Figure 5. This figure shows that the peaks for 'perpendicular polarization' are quite red-shifted compared to those generated by 'parallel polarization'. There is evidence of additional multimodal peaks in both instances. The nature of the electric fields associated with these LPSRs is discussed later.

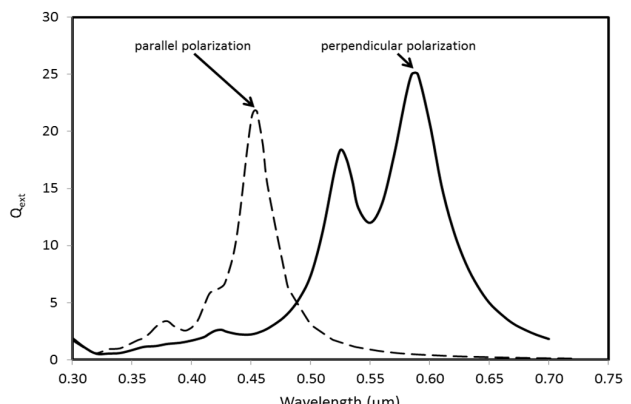

Figure 5. The calculated extinction spectra for incident light with 'parallel polarization' and 'perpendicular polarization' of a dimer of base-to-base triangles with $4.7 \mathrm{~nm}$ gap and no substrate.

The extinction spectrum is also quite dependent on the edge length, thickness and truncation (if any) of the nanotriangles but is more the aspect ratio that controls this than the actual size of the triangles. This is shown in Figure 6 . In panel (a) the extinction efficiency of two different thicknesses of base-to-base triangles of the same edge length were simulated. In panel (b) we show simulations for the two different edge lengths but the same aspect ratio. It is clear that increasing the aspect ratio will shift the dipole resonance to longer wavelengths whereas scaling the target in size mostly just scales its Qext. The gap in these simulations was $5 \mathrm{~nm}$ and there is no substrate. 
(a)

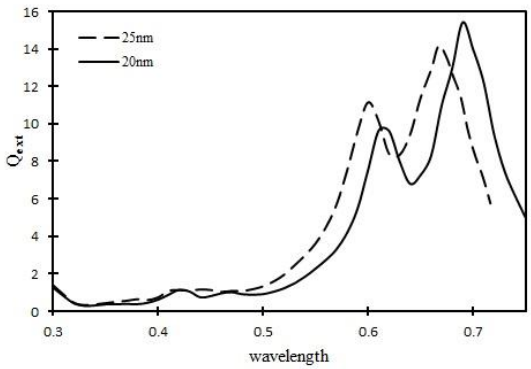

(b)

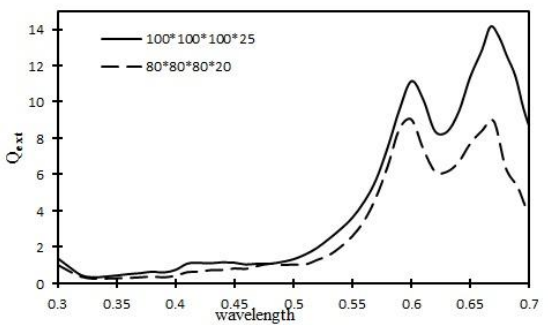

Figure 6. Effect of size and aspect ratio on the position and intensity of the LSPRs. (a) The calculated extinction spectra for $100 \times 100 \times 100 \times 20 \mathrm{~nm}$ (solid line) and $100 \times 100 \times 100 \times 25 \mathrm{~nm}$ (dashed line) base-to-base silver nanotriangles. (b) The calculated extinction spectra for $100 \times 100 \times 100 \times 25 \mathrm{~nm}$ (solid line) and $80 \times 80 \times 80 \times 20 \mathrm{~nm}$ (dashed line) base-to-base silver nanotriangles.

Next, we systematically investigated varying the gap distances from 0 to $14 \mathrm{~nm}$ in pairs of $80 \times 80 \times 80 \times 20 \mathrm{~nm}$ triangles subjected to 'perpendicular polarization', Figure 7. (Inclusion of a glass substrate caused a massive increase in the size and time of the calculations; therefore the substrate was left out in these calculations.) All other parameters were kept constant. Two main absorption bands, labelled $A$ and $B$, are observed in these spectra, and both are strongly affected by the gap size. We will show shortly that resonance $A$ starts as an ordinary dipole resonance for a gap of $0 \mathrm{~nm}$, and mutates to a complex multipole resonance as the gap is opened. Resonance $B$ requires a gap $>3 \mathrm{~nm}$ to manifest and develops into a complex coupled dipole. Both $A$ and $B$ are blue-shifted by increasing the gap distance. The shift of the LSPRs to shorter wavelengths of light is due to the decrease in mutual interaction of each triangle's charge across the gap, which gets weaker as the gap increases ${ }^{26}$ 

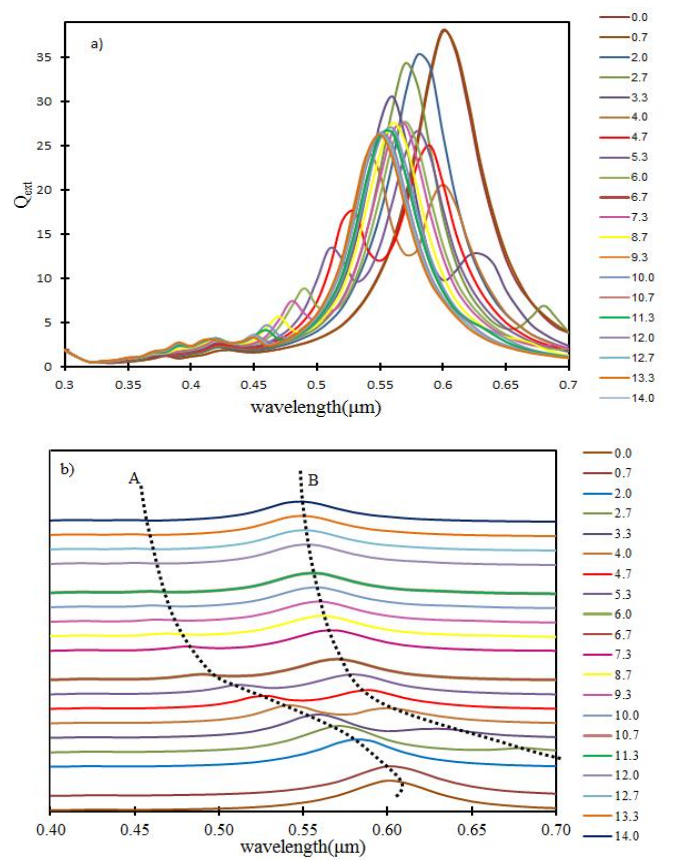

Figure 7. Calculated extinction efficiencies of $80 \times 80 \times 80 \times 20 \mathrm{~nm}$ silver nanotriangles in base-to-base configurations with polarization perpendicular to the gap. (a) plot showing values of $\mathrm{Q}_{\text {ext }}$ achieved, (b) stacked plot showing how LSPRs are red-shifted as the gap closes.

Examination of the calculated extinction spectra as a function of the gap distance showed that both the intensity and wavelength of the main resonances appear is controlled by the size of the gap. The effect is summarized in Figure 8 . There is a special situation at a gap size in the range of 4 to $5 \mathrm{~nm}$. In this case the two LSPRs are of approximately equal strength, Figure 8(a). The blue-shift in the LPSR energy as the gap is increased is summarized in Figure 8(b), however, for gaps of greater than $10 \mathrm{~nm}$ there is little further change in LSPR intensity or position. In these instances it is clear that the two triangles are sufficiently far apart to have become essentially decoupled. 

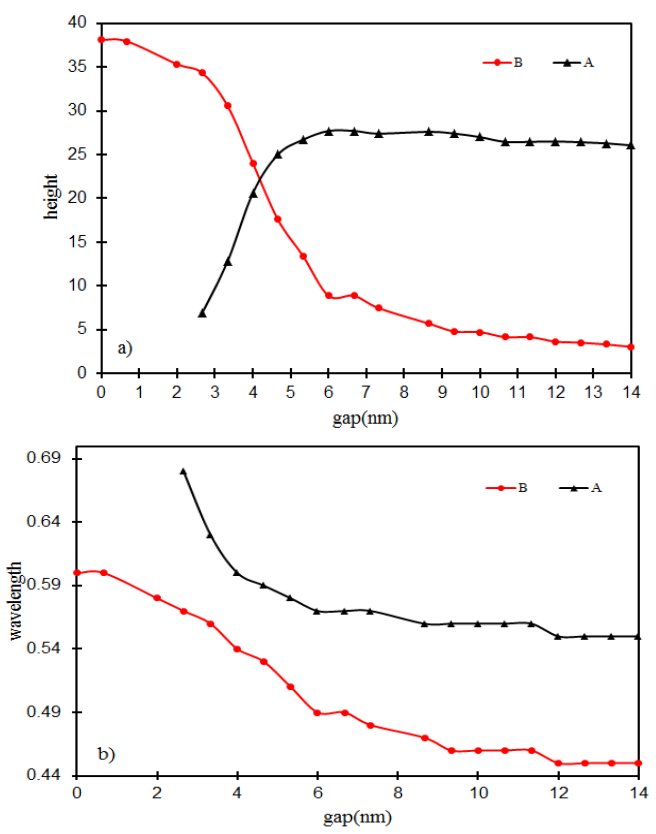

Figure 8. a) Calculated extinction efficiencies of two resonances for a pair of $80 \times 80 \times 80 \times 20 \mathrm{~nm} \mathrm{Ag}$ nanotriangles in base-tobase configuration, as a function of the gap size. b) Intensity and wavelength of the $A$ and $B$ resonances vs. gap distance.

The special situation at a gap of $4.7 \mathrm{~nm}$ is examined further in Figure 9, in which the out-of-plane component of the electric fields have been visualized on a planar section positioned about $2 \mathrm{~nm}$ above the top surface of the triangles. In respect of the case where polarization is perpendicular to the gap, there is a coupled dipolar mode at $590 \mathrm{~nm}$ (so called because the individual dipoles on the two triangles are arranged head-to-tail) and a quadrupolar mode at $530 \mathrm{~nm}$. Furthermore, if a strong field between the triangle bases is required, then the quadrupolar mode is clearly the superior choice for this gap size. The quadrupolar resonance at $530 \mathrm{~nm}$ shows a further interesting feature: two null points along the base where the polarity of the charge reverses. In the case of 'parallel polarization', the strongest resonance at 460 $\mathrm{nm}$ is simply a dipolar oscillation, but there is also an interesting high energy multimodal resonance at $360 \mathrm{~nm}$. 


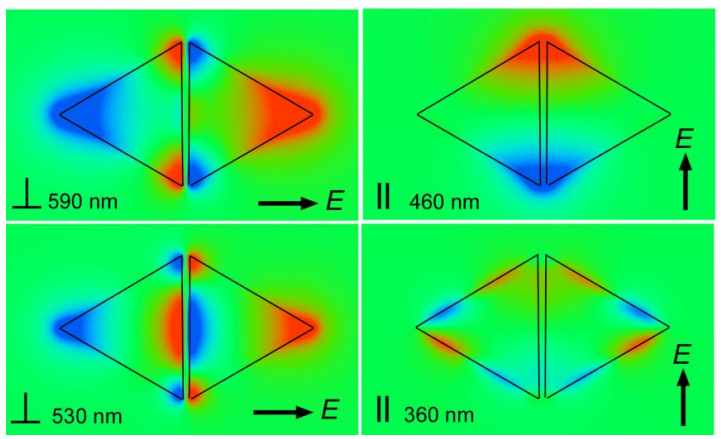

Figure 9. Simulated electric field enhancement for a pair of $80 \times 80 \times 80 \times 20 \mathrm{~nm}$ silver triangles in base-to-base configuration with a $4.7 \mathrm{~nm}$ gap. Perpendicular polarization is shown as $\perp$, parallel polarization as II. $(\lambda=590 \mathrm{~nm}$, coupled dipole mode across gap, $\lambda=460 \mathrm{~nm}$, ordinary dipole, $\lambda=530 \mathrm{~nm}$, quadrupole mode across gap, and $\lambda=360 \mathrm{~nm}$, high energy multimode.)

The electric field may also be studied by visualizing the field lines. Figure 10 shows the field lines for the case for perpendicular polarization of a pair of triangles with a $5 \mathrm{~nm}$ gap. The coupling of the charge of the dipoles on the individual triangles is clearly evident.

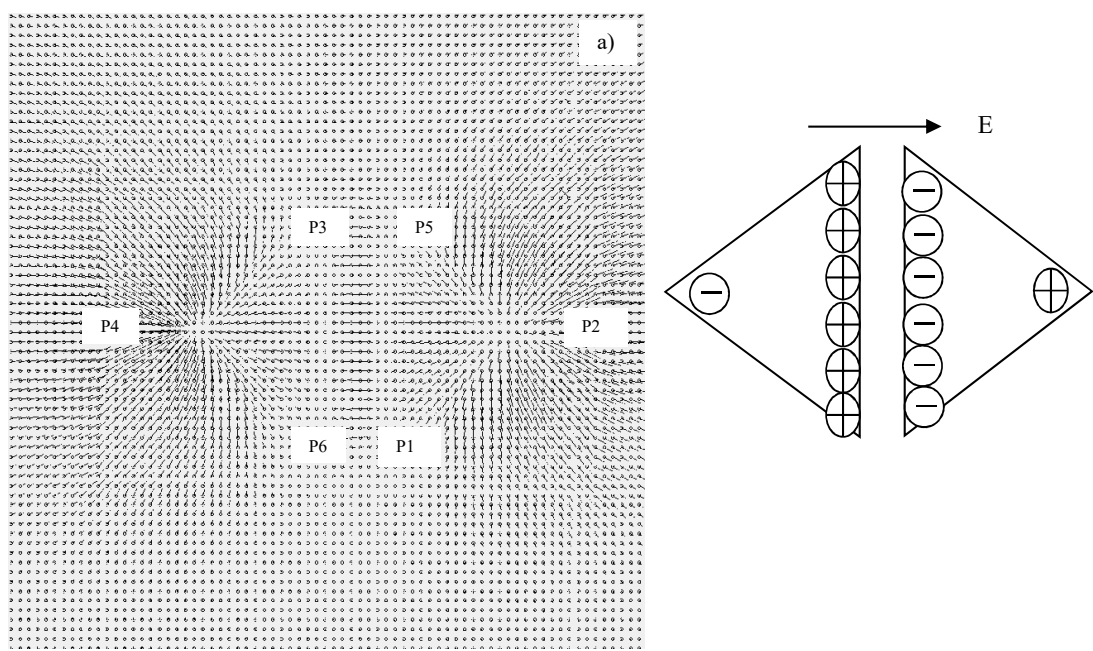

Figure 10. a) Electric field lines for dipole resonances at $\lambda=640 \mathrm{~nm}$ and b) the schematic arrangement of charge in this mode. The similarity to the charge distribution on a parallel plate capacitor is striking.

Figure 10 shows how, at a representative instant in the plasmon oscillation, negative and positive charges accumulated at the corners P4 and P2, respectively. Charge is also spread along the opposing surfaces of the gap with some concentration at the ends, with positive charge collected on the corners P3 and P6 and negative charges on the corners P1 and P5. The opposite charge state between the two bases is essentially that of a parallel plate capacitor. Not only must this result in transient storage of energy, but the potential difference across the gap should promote some electron 
tunneling. Finally, the opposing charges will cause an electrostatic attraction between the two triangles, a factor which we suggest would be interesting to examine in some future work.

\section{CONCLUSIONS}

Silver nanotriangles may be readily produced by wet chemistry and are suitable building blocks for various plasmonic structures. Here we examine dimers of triangles in the base-to-base configuration and assess the nature of the electric fields generated by their localized surface plasmon resonances. The optical properties are explored using computational techniques. The effect of varying the gap size for a base-to-base dimer of $80 \times 80 \times 80 \times 20 \mathrm{~nm} \mathrm{Ag}$ nanotriangles is considered in detail. The results indicate that the base-to-base configuration can sustain strong multimode resonances, in particular dipole and quadrupole resonances. The pairing of the parallel basal edges produces a strongly capacitive configuration and very intense electric fields over an extended volume of space during a dipolar resonance. This situation could be suitable for plasmonic devices such as sensors based on metal-enhanced fluorescence. The electric field distribution in the quadrupole resonance is more complex but may also be suitable in some instances for the excitation of fluorophores. Simulation of the extinction spectra as a function of gap space showed that both the wavelengths and intensities at which the dipole and quadrupole resonances occur are significantly influenced by gap distance.

\section{ACKNOWLEDGEMENT}

This work has been supported by Australian Research Council under DP 120102545.

\section{REFERENCES}

[1] S. A. Maier and H. A. Atwater, "Plasmonics: Localization and guiding of electromagnetic energy in metal/dielectric structures," J. Appl. Phys. vol. 98, 011101, (2005).

[2] C. J. Murphy, A. M. Gole, J. W. Stone, P. N. Sisco, A. M. Alkilany, E. C. Goldsmith and S. C. Baxter, "Gold nanoparticles in biology: Beyond toxicity to cellular imaging," J. Acc. Chem. Res. vol. 41, 1721-1730, (2008).

[3] H. Wang, D. W. Brandl, F. Le, P. Nordlander and N. J. Halas, "Nanorice: A hybrid plasmonic nanostructure," Nano Lett. vol. 6, 827-832, (2006).

[4] D. Pissuwan, S. M. Valenzuela and M. B. Cortie, "Therapeutic possibilities of plasmonically heated gold nanoparticles," Trends Biotechnol. vol. 24, 62-67, (2006).

[5] P. H. C. Camargo, L. Au, M. Rycenga, W. Li and Y. Xia, "Measuring the sers enhancement factors of dimers with different structures constructed from silver nanocubes," J. Chem. Phys. Lett. vol. 484, 304-308, (2010).

[6] M. Rycenga, M. H. Kim, P. H. C. Camargo, C. Cobley, Z.-Y. Li and Y. Xia, "Surface-enhanced raman scattering: Comparison of three different molecules on single-crystal nanocubes and nanospheres of silver $\dagger$," J. Phys. Chem. A. vol. 113, 3932-3939, (2009).

[7] B. Tang, M. Zhang, Y. Yao, L. Sun, J. Li, S. Xu, W. Chen, W. Xu and X. Wang, "Photoinduced reversible shape conversion of silver nanoparticles assisted by tio 2," J. Phys. Chem. Chem. Phys. vol. 16, 21999-22007, (2014).

[8] E. Hao and G. C. Schatz, "Electromagnetic fields around silver nanoparticles and dimers," J. Chem. Phys. vol. 120, 357-366, (2004).

[9] M. Rang, A. C. Jones, F. Zhou, Z.-Y. Li, B. J. Wiley, Y. Xia and M. B. Raschke, "Optical near-field mapping of plasmonic nanoprisms," Nano Letters. vol. 8, 3357-3363, (2008).

[10] Jiang, Zeng and Yu, "Thiol-frozen shape evolution of triangular silver nanoplates," Langmuir. vol. 23, 22182223, (2007).

[11] S. S. Shankar, A. Rai, B. Ankamwar, A. Singh, A. Ahmad and M. Sastry, "Biological synthesis of triangular gold nanoprisms," j. Nat. Mater. vol. 3, 482-488, (2004).

[12] P. R. Sajanlal and T. Pradeep, "Electric-field-assisted growth of highly uniform and oriented gold nanotriangles on conducting glass substrates," j. Adv. Mat. vol. 20, 980-983, (2008). 
[13] J. Boneberg, J. König-Birk, H. J. Münzer, P. Leiderer, K. L. Shuford and G. C. Schatz, "Optical near-fields of triangular nanostructures," Appl. Phys. A. vol. 89, 299-303, (2007).

[14] N. Félidj, J. Grand, G. Laurent and J. Aubard, "Multipolar surface plasmon peaks on gold nanotriangles," J. Chem. Phys. vol. 128, 094702, (2008).

[15] N. Yu, E. Cubukcu, L. Diehl and D. Bour, "Bowtie plasmonic quantum cascade laser antenna," J. Opt. Express. vol. 15, 13272-13281, (2007)

[16] L. Yang, C. Du and X. Luo, "Numerical study of optical properties of single silver nanobowtie with anisotropic topology," J. Appl. Phys. B. vol. 92, 53-59, (2008).

[17] R. Jin, Y. Cao, C. A. Mirkin, K. L. Kelly and G. C. Schatz, "Photoinduced conversion of silver nanospheres to nanoprisms," Science. vol. 294, 1901-1903, (2001).

[18] D. E. Charles, D. Aherne, M. Gara, D. M. Ledwith, Y. K. Gun'ko, J. M. Kelly, W. J. Blau and M. E. BrennanFournet, "Versatile solution phase triangular silver nanoplates for highly sensitive plasmon resonance sensing," J. ACS Nano. vol. 4, 55-64, (2009).

[19] L. J. Sherry, R. Jin, C. A. Mirkin, G. C. Schatz and R. P. Van Duyne, "Localized surface plasmon resonance spectroscopy of single silver triangular nanoprisms," J. Nano Lett. vol. 6, 2060-2065, (2006).

[20] K. L. Kelly, E. Coronado, L. L. Zhao and G. C. Schatz, "The optical properties of metal nanoparticles: The influence of size, shape, and dielectric environment," J. Phys. Chem. B. vol. 107, 668-677, (2003).

[21] M. Moskovits, "Surface-enhanced Raman spectroscopy: A brief retrospective," J. Raman Spectrosc. vol. 36, 485-496, 2(005)

[22] M. Tsuji, Y. Maeda, S. Hikino, H. Kumagae, M. Matsunaga, X.-L. Tang, R. Matsuo, M. Ogino and P. Jiang, "Shape evolution of octahedral and triangular platelike silver nanocrystals from cubic and right bipyramidal seeds in dmf," J. Cryst. Growth Des. vol. 9, 4700-4705, (2009).

[23] B. T. Draine and P. J. Flatau, "Discrete-dipole approximation for scattering calculations," J. JOSA A. vol. 11, 1491-1499, 1994

[24] B. T. Draine and P. J. Flatau, "Discrete-dipole approximation for scattering calculations," J. Opt. Soc. Am. A. vol. 11, 1491-1499, (1994)

[25] N. Shahcheraghi, A. Dowd, A. McDonagh, M. B. Cortie and V. Keast, "Multimode plasmon resonances on double- and triple-decker stacks of silver nanotriangles," in Nanoscience and Nanotechnology (ICONN), 57-60 (2014).

[26] S. Dodson, M. Haggui, R. Bachelot, J. Plain, S. Li and Q. Xiong, "Optimizing electromagnetic hotspots in plasmonic bowtie nanoantennae," J. Phys. Chem. Lett. vol. 4, 496-501, (2013) 